\title{
DIAGNOSTIC ACCURACY OF C-14 UREA BREATH TEST FOR DETECTION OF HELICOBACTER PYLORI IN PATIENTS WITH GASTRITIS
}

\author{
Zaigham Salim Dar, Suniya Arshad Butt*, Khurram Hayat Mir*, Fida Hussain \\ Armed Forces Institute of Pathology/National University of Medical Sciences (NUMS) Rawalpindi Pakistan, *PAF Hospital, Mianwali Pakistan
}

\begin{abstract}
Objective: To evaluate the diagnostic accuracy of C-14 Urea Breath Test for detection of helicobacter pylori infection in patients with gastritis.

Study Design: Cross-sectional validation study.

Place and Duration of Study: Nuclear Medical Centre, Armed Forces Institute of Pathology, from Feb to Aug 2016.

Methodology: After fulfilling inclusion and exclusion criteria, 110 patients of both genders, aged between 18-50 years, were enrolled. Prior written informed consent was obtained from each patient. First, they were assessed by C-14 Urea Breath Test, followed by endoscopic biopsy and histopathology. Results of C-14 Urea Breath Test were compared to histopathology diagnosis which was taken as gold standard.

Results: Patients' ages ranged from 18-50 years with a mean \pm SD of $37.45 \pm 10.21$ years. Seventy four (67.3\%) of them were males and $36(32.7 \%)$ were females. Sixty four (58.2\%) patients were suspected of helicobacter pylori on C-14 Urea breath test. However, histopathology of endoscopic biopsy confirmed helicobacter pylori in 66 (60\%) patients yielding 64 true positive, 44 true negative and 2 false negative cases. Calculated sensitivity was $96.97 \%$, specificity $100 \%$ and accuracy was $98.18 \%$ for C- 14 Urea Breath Test with negative and positive predictive values of $95.65 \%$ and $100 \%$ respectively.

Conclusion: C-14 Urea Breath Test is highly accurate, sensitive and specific test for detection of helicobacter pylori infection, irrespective of patient's age and gender.
\end{abstract}

Keywords: C-14 urea breath test, Endoscopic biopsy, helicobacter pylori, Histopathology.

\footnotetext{
This is an Open Access article distributed under the terms of the Creative Commons Attribution License (https://creativecommons.org/licenses/by-nc/4.0/), which permits unrestricted use, distribution, and reproduction in any medium, provided the original work is properly cited.
}

\section{INTRODUCTION}

Helicobacter pylori (H. pylori) is a gram negative spiral-shaped bacterium which thrives in the digestive tract. H. pylori infection has a very high prevalence worldwide, and may be found in more than half of the world's population ${ }^{1}$. The organism grows in the acidic environment of the gastric mucosa. It has high urease activity which can convert urea present in the gastric mucosa to carbon dioxide and ammonia ${ }^{2}$.

Traditionally $H$. pylori infectionis diagnosed with non-invasive as well as invasive techniques. Invasive methods include polymerase chain reaction (PCR), culture and histopathology of the endoscopic biopsy specimen $^{3}$. However, non-invasive tests are the first line of action for diagnosing H. pylori infection. Among these, stool antigen test, serological tests, and urea breath test are the most frequently used ${ }^{4}$.

C-14 labeled urea is administered orally, where it reacts with bacterial Urease enzyme produced by H. pylori inside patients' stomach. Radiolabelled urea is hydrolyzed, producing radio labeled $\mathrm{CO} 2$, which is absorbed into the bloodstream and is then exhaled

Correspondence: Dr Zaigham Salim Dar, Consultant Nuclear Medicine, Armed Forces Institute of Cardiology, Rawalpindi Pakistan Received: 13 May 2020; revised received: 22 Jun 2020; accepted: 29 Jun 2020 from the lung. The activity of radiolabeled $\mathrm{CO} 2$ can be measured in the breath sample ${ }^{5}$.

Being highly sensitive and specific as well noninvasive test, Urea Breath Test is very attractive procedure for microbiologists and clinicians for detection of $H$. pylori infections especially in asymptomatic patients where Urea Breath Test is considered a gold standard. Adding to its advantages is the fact that Urea Breath Test is free of sampling errors which are more commonly encountered in case ofendoscopic biopsy. This superiority makes it very popular among clinicians, especially in asymptomatic, elderly, and pediatric subjects ${ }^{6}$.

Considering high prevalence of the disease and economic constraint in developing countries like ours, use of C-14 Urea Breath Testis recommended over invasive procedures like endoscopic biopsy and histopathology ${ }^{7}$.

The study objective was to evaluate the diagnostic accuracy of C-14 Urea Breath Testagainst culture and histopathology of endoscopic biopsy. The rationale behind this study was to evaluate C-14 Urea Breath Test in our setup and to subsequently recommend it as an accurate, non-invasive, sensitive and specific test for H. pylori detection in routine management of patients of chronic gastritis. 


\section{METHODOLOGY}

This research project was carried out at Armed Forces Institute of Pathology, Rawalpindi's Nuclear Medical Centre, from February to August 2016. Sample size $(n=110)$ was calculated by using WHO sample size calculator with confidence interval $95 \%$, Power of test $80 \%$, level of significance $5 \%$, Anticipation of the population proportion $(\mathrm{P} 0)=0.9$ (sensitivity of urea breath test test $>90 \%$ ) and prevalence of $50 \%{ }^{1}$. Through non probability consecutive sampling, 110 patients were included in the study, after fulfilling the inclusion and exclusion criteria. Patients with acid peptic disease, aged 18-50 years, were included in the study that had no history of antibiotic, Proton pump inhibitors or H2 receptor blocker treatment in last 2 weeks. Patients on chronic NSAID, steroid or immunosuppressive therapy were excluded. Patients with any history of gastric procedure in the past were also excluded. Pregnant females were not included in the study.

The study was conducted after approval from hospital ethics committee (Armed Forces Institute of Pathology Ethical Review Committee approval signed 20 Jan 2016). Eligible patients were enrolled in trial after taking informed consent. For C-14 Urea Breath Test, patients were asked to fast overnight. Heliprobe ${ }^{\circledR}$ system was employed for conduction of the test. Patients had to swallow a capsule containing $1 \mu \mathrm{CiC}-14$ labeled urea with $20 \mathrm{ml}$ of lukewarm water. After 5 min, patient again had to take $20 \mathrm{ml}$ of lukewarm water. At 10 min of radiopharmaceutical administration, the patient was instructed to take a deep breath and hold it for almost 5-10 sec. Patient then exhaled into a Mylar balloon through a straw. Liquid scintillation counter was used to analyze the breath sample in C-14 $\beta$ particles window. Activity was measured in disintegration per minute (dpm). Patient was declared positive if breath samples had a count rate of $200 \mathrm{dpm}$ and negative when the count rate was $<50 \mathrm{dpm}$. However, the indeterminate cases having count rate of 50$199 \mathrm{dpm}$ were also declared negative for the sake of study. Diagnostic criterion is described in table-I.

Table-I: Diagnostic criteria.

\begin{tabular}{l|c}
\hline \multicolumn{2}{c}{ Diagnostic Criteria } \\
\hline Negative & $<50 \mathrm{dpm}$ at $10 \mathrm{~min}$ \\
\hline Indeterminate & $<50-200 \mathrm{dpm}$ at $10 \mathrm{~min}$ \\
\hline Positive & $>200 \mathrm{dpm}$ at $10 \mathrm{~min}$ \\
\hline
\end{tabular}

Later on gastric endoscopy was performed and 2 gastric biopsy specimen were obtained, one from the antrum and other from corpus. Both of the samples were studied under standard hematoxilin and eosin and giemsa stains for histopathology. The result was considered positive if $H$. pylori was detected in any one of the stain and declared negative if both specimen showed no growth.

SPSS-20 was used to analyze all the collected data. Numerical variables like age were presented as mean \pm $\mathrm{SD}$. Categorical variables like gender and presence of H. pylori on C-14 urea breath test and histopathology were presented as frequency and percentage. A $2 \times 2$ contingency table was generated to calculate sensitivity, specificity, positive and negative predictive values and accuracy of C-14 Urea Breath Test for diagnosis of $H$. pylori infection keeping the histopathology diagnosis as standard.

\section{RESULTS}

A total of 110 patients were included in the study with their ages ranging from 18-50 years having mean $\pm \mathrm{SD}$ of $37.45 \pm 10.21$ years. Sixty eight $(61.8 \%)$ patients were aged between 18-34 years while rest of the 42 $(38.2 \%)$ were aged between $35-50$ years. Seventy four $(67.3 \%)$ of the patients were male and remaining 36 $(32.7 \%)$ were female.

Sixty foru (58.2\%) patients diagnosed as positive for H. pylori infection on C14 Urea Breath Test were confirmed to be positive on histopathology of the endoscopic biopsy. However, out of 46 (42.8\%) patients labeled H. pylori negative by C14 Urea Breath Test, 44 were confirmed as negative by histopathology of endoscopic biopsy while 2 were diagnosed as positive for infection. Therefore, 64 cases came out to be true positive, 44 true negative and 2 were false negative. None of the cases was false positive. C14 Urea Breath Test, therefore, yielded $96.97 \%$ sensitivity, $100 \%$ specificity, $98.18 \%$ diagnostic accuracy along with negative and positive predictive values of $95.65 \%$ and $100 \%$ respectively. The results are shown in table-II \& III.

Table-II: Comparison of urea breath test and histopathology.

\begin{tabular}{|c|c|c|c|c|}
\hline & \multicolumn{2}{|c|}{ Histopathology } & \multirow{2}{*}{ Total } \\
\hline & & Positive & Negative & \\
\hline \multirow{2}{*}{$\begin{array}{l}\text { Urea } \\
\text { Breath Test }\end{array}$} & Positve & $64(\mathrm{TP})$ & $0(\mathrm{FP})$ & 64 \\
\hline & Negative & $2(\mathrm{FN})$ & $44(\mathrm{TN})$ & 46 \\
\hline \multicolumn{2}{|c|}{ Total } & 66 & 44 & 110 \\
\hline \multicolumn{5}{|c|}{ Table-III: Diagnostic parameters. } \\
\hline \multicolumn{2}{|c|}{ Diagnostic Parameters } & \multicolumn{2}{|c|}{ Formula } & Value \\
\hline \multicolumn{2}{|l|}{ Sensitivity } & \multicolumn{2}{|c|}{$\mathrm{TP} /(\mathrm{TP}+\mathrm{FN})$} & $96.97 \%$ \\
\hline \multicolumn{2}{|l|}{ Specificity } & \multicolumn{2}{|c|}{$\mathrm{TN} /(\mathrm{TN}+\mathrm{FP})$} & $100 \%$ \\
\hline \multicolumn{2}{|c|}{ Positive Predictive Value } & \multicolumn{2}{|c|}{$\mathrm{TP} /(\mathrm{TP}+\mathrm{FP})$} & $98.18 \%$ \\
\hline \multicolumn{2}{|c|}{ Negative Predictive Value } & \multicolumn{2}{|c|}{$\mathrm{TN} /(\mathrm{TN}+\mathrm{FN})$} & $95.65 \%$ \\
\hline \multicolumn{2}{|c|}{ Diagnostic Accuracy } & \multicolumn{2}{|c|}{$(\mathrm{TP}+\mathrm{TN}) / \mathrm{n}$} & $100 \%$ \\
\hline
\end{tabular}




\section{DISCUSSION}

H.pylori is a gram-negative pathogen living in human gastric mucosa. Without the specific antibiotic treatment, this infection can cause chronic active gastritis which can subsequently leads to peptic ulcer in 15$20 \%$ of the patients and gastric malignancy and mucosa-associated lymphoid tissue (MALT) lymphomain approximately $1-3 \%$. Chronic H.pylori infection has been identified as one of the most important etiological factors for gastric cancer which is third leading cause of cancer death globally ${ }^{8}$. Javed et al, have reported prevalence of $H$. Pylori infection $92 \%$ among patients of gastritis 9 . A recently published systematic review and meta-analysis by Hooi et al, classified Pakistan among countries with highest $H$. pylori prevalence $(81 \%)^{10}$.

Presently a lot of methods are employed to detect the presence of $H$. pylori. While all of them have their own limitations, disadvantages and advantages, there is a general division of the available tests into invasive and non-invasive tests. Invasive techniques need gastro-endoscopy and captivating gastric biopsy specimens for histopathology, culture and rapid urease activity. Other category is of noninvasive tests use peripheral samples, like as stools, blood and breath samples to be used in detecting antibodies, urease activity or bacterial antigens ${ }^{11}$.

Specificity and the sensitivity of histopathology for $H$. pylori diagnosis varies from $53-90 \%$, based upon the density of colonization and pathologist's experience, while that of rapid urease test (RUT) ranges from $95-100 \%$ and $85-95 \%$ respectively. However, for the detection of $H$. pylori, culture is used as a gold standard method with specificity $100 \%$ despite the fact that in various laboratories it has variable sensitivity to a considerable level ${ }^{12}$.

For identification of antibodies against H. pylori, a huge number of tests have been used. Most of the kits are based on enzyme immunoassay (EIA) and immunoblot (IM) as major immunological techniques. Advantages of serological tests include easy availability, low cost, and easy to perform methodology. They should be given priority in patients with bleeding ulcers, gastric atrophy or those who had recently used antibiotics or proton pump inhibitors ${ }^{12}$.

Antigen detection in stool assay is the most recent non-invasive techniques. $H$. pylori antigens that are excreted in stool are detected by ELISA using either monoclonal or polyclonal antibodies ${ }^{13}$. Gisbert et al, (2006) evaluated the stool antigen detection technique and found the specificity and sensitivity of $97 \%$ and $94 \%$, repectively ${ }^{14}$.

$\mathrm{C} 14$ is a $\beta$-emitting radioactive isotope of carbon, with maximum $\beta$-energy of $156 \mathrm{keV}$ and half-life of 5730 years. It is measured in a liquid scintillation counter. Standard oral dose of $1 \mu$ Cidelivers an estimated radiation dose of $\langle 3 \mu \mathrm{Sv}$, which is negligible when compared to routine radiological procedures which, on average produce radiation burden of 5-30 mSvin each study. However, even this meagre radiation dose can be avoided by using newer C-13 based Urea Breath Test. C-13 is a stable isotope of Carbon which is measured by mass spectrometer instead of any scintillation detector ${ }^{15}$. Charets et al, compared C-13 and C-14 Urea Breath Test and found that sensitivity of C-13 Urea Breath Test ranged from $89-97 \%$, while that of C-14 urea breath test ranged from $89-94 \% 16$. However, other study has shown that C-13 urea breath test provides clearer positive or negative results, with fewer borderline results as compared to C-14 urea breath test ${ }^{15}$.

In our study, patients aged from 18-50 years with a mean \pm SD of $37.45 \pm 10.21$ years. Aftab et al, and Zhou et al. have reported similar mean ages, of $34.2 \pm$ 11.6 years and $36 \pm 11$ years, in Bangladeshi and Chinese populations respectively ${ }^{17,18}$. However, relatively higher mean age has been reported previously by Mehmood et al, (45 \pm 6.3 years) and Yakoob et al. (41 \pm 13 years) in Pakistan ${ }^{19,20}$. There were $74(67.3 \%)$ male and $36(32.7 \%)$ female patients in our study with a male to female ratio of 2.1:1. A similar ratio of 2:1 has been reported previously by Yakoob et al, in another local study ${ }^{20}$. Many other authors have also reported male predominance; Mehmood et al. (64.9\% vs. $35.1 \%)$ and Rasheed et al. $(51.9 \% \text { vs. } 48.1 \%)^{19,21}$.

Thsi study yielded $96.97 \%$ sensitivity, $100 \%$ specificity and $98.18 \%$ accuracy for C-14 Urea Breath Test fort the diagnosis of $H$. pylori with negative and positive predictive values of $95.6 \%$ and $100 \%$ respectively. Pooled results of a recent meta-analysis by Zhou et $a l$, comprising 18 studies, indicated that the C-14 Urea Breath Test showed a diagnostic sensitivity of 0.96 (0.95-0.96 at $95 \% \mathrm{CI})$ and specificity of 0.93 (0.91-0.94 at $95 \% \mathrm{CI})^{22}$. Özdemir et al, also reported $96.6 \%$ sensitivity, $100 \%$ specificity, $97.7 \%$ accuracy, $100 \%$ positive predictive value and $93.7 \%$ negative predictive values for C-14 Urea Breath Test ${ }^{23}$.

Thabit et al, reported similar specificity of $100 \%$ but with very low sensitivity of $81.6 \%$, as compared to the present study. Rasool et al, on the other hand, 
reported similar high sensitivity of $98 \%$ but very low specificity of $91 \% 24$.

\section{CONCLUSION}

C-14 Urea Breath test was found to be $96.97 \%$ sensitive, $100 \%$ specific and $98.18 \%$ accurate for the diagnosis of $H$. pylori with negative and positive predictive values of $95.65 \%$ and $100 \%$ respectively. The diagnostic performance of C-14 Urea Breath Test was unaffected by patient's age and gender. This high sensitivity and specificity of C-14 Urea Breath Test, along with its non-invasive technique and low cost, make it an ideal screening and diagnostic tool.

\section{CONFLICT OF INTEREST}

This study has no conflict of interest to be declared by any author.

\section{REFERENCES}

1. Diaconu S, Predescu A, Moldoveanu A, Pop CS, FierbineanuBraticevici C. Helicobacter pylori infection: old and new. J Med Life 2017; 10(2): 112-17.

2. Ferwana M, Abdulraajeed I, Alhajiahmad A, Madani W, Firwana B, Hassan R, et al. Accuracy of urea breath test in Helicobacter pylori infection, Meta-analysis. World J Gastroenterol 2015; 21(4): 1305-14.

3. Filomena A, Guenther A, Planatscher H, Topin F, She J, Formichella L, et al. Performance of a multiplex serological helicobacter pylori assay on a novel microfluidic assay platform. Proteom 2017; 5(4): 24-28.

4. Kayali S, Aloe R, Bonaguri C, Gaiani F, Manfredi M, Leandro G, et al. Non-invasive tests for the diagnosis of helicobacter pylori: state of the art. Acta bio-medica: Atenei Parmensis 2018; 89 (Suppl8): 58-64.

5. Graham DY, Miftahussurur M. Helicobacter pylori urease for diagnosis of Helicobacter pylori infection: A mini review. Jadv Res 2018; 13(9): 51-57.

6. Abadi ATB. Diagnosis of Helicobacter pylori Using Invasive and Noninvasive Approaches. J Pathog 2018; 2018(2): 9064952-55.

7. Rasheed F, Yameen A, Ahmad T, Bilal R. Rate of active helicobacter pylori infection among symptomatic patients of Pakistan. Malaysian J Pathol 2017; 39(1): 69-72.

8. Li H, Yang TK, Tang H, Tang X, Shen Y, Benghezal M, et al. Helicobacter pylori infection is an infectious disease and the empiric therapy paradigm should be changed. Precision Clin Med 2019; 2(2): 77-80.

9. Javed M, Amin K, Muhammad D, Husain A, Mahmood N. Prevalence of H. Pylori. Professional Med J 2010; 17(3): 431-39.

10. Hooi JKY, Lai WY, Ng WK, Suen MMY, Underwood FE, Tanyingoh D, et al. Global Prevalence of Helicobacter Pylori infection:
Systematic review and Meta-Analysis. Gastroenterol2017; 153(2): 420-29.

11. Megraud F, Lehours P. Helicobacter pylori detection and antimicrobial susceptibility testing. Clin Microbiol Rev 2007; 20(2): 280-22.

12. Garza-Gonzalez E, Perez-Perez GI, Maldonado-Garza HJ, Bosques-Padilla FJ. A review of Helicobacter pylori diagnosis, treatment, and methods to detect eradication. World J Gastroenterol 2014; 20(6): 1438-49.

13. Deguchi R, Matsushima M, Suzuki T, Mine T, Fukuda R, Nishina $\mathrm{M}$, et al. Comparison of a monoclonal with a polyclonal antibody-based enzyme immunoassay stool test in diagnosing Helicobacter pylori infection after eradication therapy. J Gastroenterol 2009; 44(7): 713-16.

14. Gisbert JP, de la Morena F, Abraira V. Accuracy of monoclonal stool antigen test for the diagnosis of $\mathrm{H}$. pylori infection: a systematic review and meta-analysis. Am J Gastroenterol 2006; 101(8): 1921-30.

15. Charets M, Belair MA. Comparison of Accuracy Between 13Cand 14 C-Urea Breath Testing: Is an Indeterminate-Results Category Still Needed? J Nucl Med Technol 2017; 45(2): 87-90.

16. Best LM, Takmoingi $Y$, Siddique S, Selladurai A, Gandhi A, Low $B$, et al. Non- invasive diagnostic tests for Helicobacter pylori infection. Cochrane Database Syst Rev 2018; 3(3): CD012080.

17. Aftab H, Miftahussurur M, Subsomwong P, Ahmed F, Khan AA, Yamaoka Y. Helicobacter pylori antibiotic susceptibility patterns in Bangladesh: Emerging levofloxacin resistance. J Infect Dev Ctries 2016; 10(3): 245-53.

18. Zhou YQ, Xu L, Wang BF, Fan XM, Wu JY, Wang CY, et al. Modified sequential therapy regimen versus conventional triple therapy for helicobacter pylori eradication in duodenal ulcer patients in China: a multicenter clinical comparative study. Gastroenterol Res Prac 2012; 2012(1): 405425-28.

19. Mehmood K, Awan AA, Muhammad N, Hasan F, Nadir A. Helicobacter pylori prevalence and histopathological findings in dyspeptic patients. J Ayub Med Coll Abbott 2014; 26(2): 182-85.

20. Yakoob J, Abid S, Jafri W, Abbas Z, Mumtaz K, Hamid S, et al. Low rate of recurrence of Helicobacter pylori infection in spite of high clarithromycin resistance in Pakistan. BMC Gastroenterol 2013; 13(1): 33-36.

21. Rasheed F, Ahmad T, Bilal R. Prevalence and risk factors of Helicobacter pylori infection among Pakistani population. Pak J Med Sci 2012; 28(4): 661-65.

22. Zhou Q, Li L, Ai Y, Pan Z, Guo M, Han J. Diagnostic accuracy of the 14C-urea breath test in Helicobacter pylori infections: a metaanalysis. Wien Klin Wochenschr 2017; 129(1-2): 38-45.

23. Ozdemir E, Karabacak NI, Degertekin B, Cirak M, Dursun A, Engin D, et al. Could the simplified $14 \mathrm{C}$ urea breath test be a new standard in noninvasive diagnosis of Helicobacter pylori infection?. Ann Nucl Med 2008; 22(7): 611-16.

24. Rasool S, Abid S, Jafri W. Validity and cost comparison of 14 carbon urea breath test for diagnosis of $\mathrm{H}$ Pylori in dyspeptic patients. World J Gastroenterol 2007; 13(6): 925-29. 